\title{
On the Counting Methods of White Blood Cells in the Peripheral Blood and Nucleated Cells in the Bone Marrow, with Special Reference to Biological Significance of the Absolute Count and the Percentage of the Leucocytes*
}

\author{
By \\ Akira Saito \\ The Medical Department of Prof. S. Y a mag a t a, Tohoku University \\ School of Medicine, Sendai
}

(Received for publication, January 20, 1964)

\begin{abstract}
The distribution of body defence energy was studied from the standpoint of the hematopoietic phases of the bone-marrow and the fields and times of blood defense reaction. It was found that the sum of energy poured into the field of cell-bacterium reaction and the field of antibody-antigen reaction is equal to the defense energy of the body, and the law of conservation of defense energy is entirely the same as Mayer-Joule-Helmholtz's law of conservation of energy. From this law, it is construed that the cell-bacterium reaction and antibodyantigen reaction show a constant-antagonistic relation. The cells playing celllbacterium reaction, especially neutrophils, show less qualitative changes but more quantitative changes in response to stimulations, and the cells playing antibodyantigen reaction, especially lymphocytes, show less quantitative changes but more qualitative changes by stimulations. Therefore, while the absolute count of leucocytes does not show the distribution of defense energy, their percentage shows the distribution of defense energy, adaptation of the body and characteristics of stimulant factors, and then the biological and clinical value of their percentage is far superior as compared with absolute count.

In order to count the nucleated cells in the bone-marrow, the generally adopted method which makes total nucleated cells as $100 \%$, is inconvenient and unreasonable from the standpoint of the hematopoietic phases in the bonemarrow and the fields and times of blood defense reaction. Rohr's counting method is excellent and reasonable as calculating method of the nucleated cells in the bone-marrow.
\end{abstract}

The hematopoietic function of the bone marrow was discussed from the standpoint of the hematopoietic phases and the fields and times of blood defense reaction in the preceding reports ${ }^{1-16}$. In these studies ${ }^{1-31)}$, while the total count,

斎藤 草

* The 17th Report on the Essential Nature of the Hematopoietic Function of Bone Marrow 
of leucocytes in the peripheral blood was given in absolute number, the differential count of leucocytes was always given in percentage, and some critical objections have been raised that the change in percentages of the differential count would indicate only a relative change, but the real picture of the hematopoiesis in the bone marrow and leucocyte reaction can be portrayed only by tracing the change in the absolute count of leucocytes.

Of course, it is needless to say that the absolute number is the means of representing the real quantitative relation with highest accuracy and directness, and a "change in absolute number", being an absolute change, is more reliable and valuable than a "relative change" in quantitative discussion, as far as the items discussed are similar in size and properties. In a case when the items comprise things perceptibly variable in size and/or properties, the absolute number would lose its preferential position.

For example, a match-stick and a totem-pole, possibly made of the same material, are incommensurate in size; a litre of water and a litre of oil are equal in quantity, but different in properties, so it may lead us to ridiculous conclusions if we take only the abolute number or quantity of the pair of objects in discussing them in comparison.

Similarly, as the erythrocytes are normally very similar in size and quality, their absolute count is a good index for detecting and following up a change in the blood picture. But as the leucocytes comparise different kinds of neutrophils, monocytes, lymphocytes, eosinophils and basophils, in addition to these, endothelial cells, plasma cells and atypical lymphocytes under stimulation, they are widely variable in function, though not much different in size $(6-12 \mu)$. So it would be rather purposeless to discuss the change in the absolute count of individual types, and it is the author's belief that the method of describing the percentage constitution of leucocytes is preferable.

The Biological Significance of Percentage and Absolute Count of Leucocytes from the Viewpoint of Hematopoietic Phases of Bone Marrow and of Fields and Times of Blood Defense Reaction

As already reported ${ }^{1-16)}$, the defense reaction of a living organism is effected through cell-bacterium reaction and antibody-antigen reaction, the former being unspecific but the latter specific to different stimulant factors, and the two reactions, acting antagonistically, are enacted in different fields, are essentially different in nature and not mutually compensatory. Of leucocytes in the peripheral blood, neutrophils, monocytes and endothel cells act in the cellbacterium reaction field, and lymphoctyes, eosinophils, basophils, plasma cells and atypical lymphocytes act in the antibody-antigen reaction field. 
1. Biological Significance of Percentage and Absolute Count of Leucocytes as Viewed from the Angles of Hematopoietic Phases of Bone Marrow and of Fields and Times of Blood Defense Reaction

A) Characteristics of cells active in the cell-bacterium reaction (phagocytosis)

Neutrophils, monocytes and, under stimulation, endothel cells come into activity in the cell-bacterium reaction disposing of the invading microbes by phagocytosis, and are called phagocytes. They are ready to act non-specifically against any pathogenic or stimulant factors, so that the defense reaction can be initiated by neutrophils at the onset of the disease, before the antibodies are produced, presenting alarm reaction (Selye $\left.{ }^{33}\right)$ ). In and after the acme, according to the specificity of the invading pathogenic factors of the I, II or III group, the neutropoietic system of the bone marrow comes into 1 st-phase, intermediate or 2nd-phase reaction, which entails a change in neutrophil count in the peripheral blood, causing leucocytosis, normo-leucocytosis or leucopenia (Fig. 1).

The cell-bacterium reaction is effected by the blood cells of the host directly taking the pathogenic agents and diposing of them by digestion, and morphologically emphasized in the host-parasite relation.

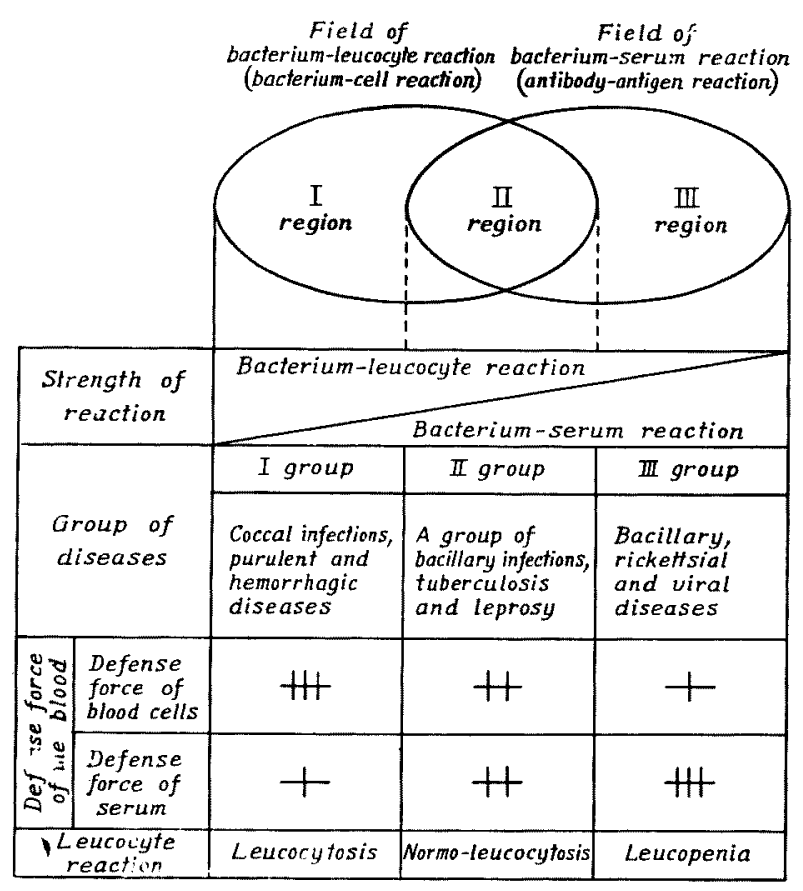

Fig. 1. Relationships between the strengths of cell-bacterium reaction, of antibodyantigen reaction, and leucocyte count in the peripheral blood. 
Here, the strength of reaction is in direct proportion to the number of phagocytes, that is, the larger the number of neutrophils, the stronger the reaction. So, as shown in Fig. 1, this reaction is strongest in diseases of leucocytotic type, next in those of normo-leucocytotic type, and weakest in those of leucopenic type. Thus, the strength of cell-bacterium reaction being dependent on the absolute number of phagocytes, the absolute number of such cells is here a more exact and valuable indicator than their percentage.

B) Characteristics of cells active in the antibody-antigen reaction (antibodyproducing cells)

Lymphocytes, eosinophils, basophils and, under stimulation, plasma cells and atypical lymphocytes cause antibody-antigen reaction by producing antibodies. Of these cells, lymphocytes, atypical lymphocytes and plasma cells probably engender antimicrobial antibodies, to act indirectly upon the pathogenic agents in the defense reaction.

The antibodies, i.e. specific biochemical substances, are ones produced by the antibody-producing cells of the host under stimulation from pathogenic agents and physico-chemical factors. Therefore, the function of these cells undergoes a drastic change upon invasion of such stimulant factors. At the time of invasion, the absolute count of these cells drops temporarily but soon begins to rise, and there is a great difference between different pathogenic microbes in their antibody-producing capacity, so that in the antibody-antigen reaction, more importance should be attached to the change in the relative number of the cells than to the change in absolute count.

The change in the relative number of the cell varieties depends on the strength of biological activity of the stimulant factors, that is, the stronger the activity, the larger the output of antibodies.

This biological activity is strongest in the Group III factors comprised of viruses, rickettsiae, typhoid bacilli and some other bacilli, less strong in the Group II germs comprised of tubercle and leprosy bacilli, and weakest in the Group I germs comprised of diplococci, staphylococci and streptococci. Accordingly, the antibody output, that is, the antibody-antigen reaction is the strongest against Group III germs, followed by that against Groups II and I, in the order named.

The biological activity of the pathogenic agents is related to the qualitative or functional change of the host cells but not directly related to their number, so that a change in the absolute number of the antibody-producing cells does not necessarily reflect the strength of the antibody-antigen reaction.

Thus, the antibody-antigen reaction is a specific reaction, the antibody output of the cells being proportionate to the biological activity of the antigen and to the appearance and rise of atypical lymphocytes. Consequently, 


\section{Percentage}
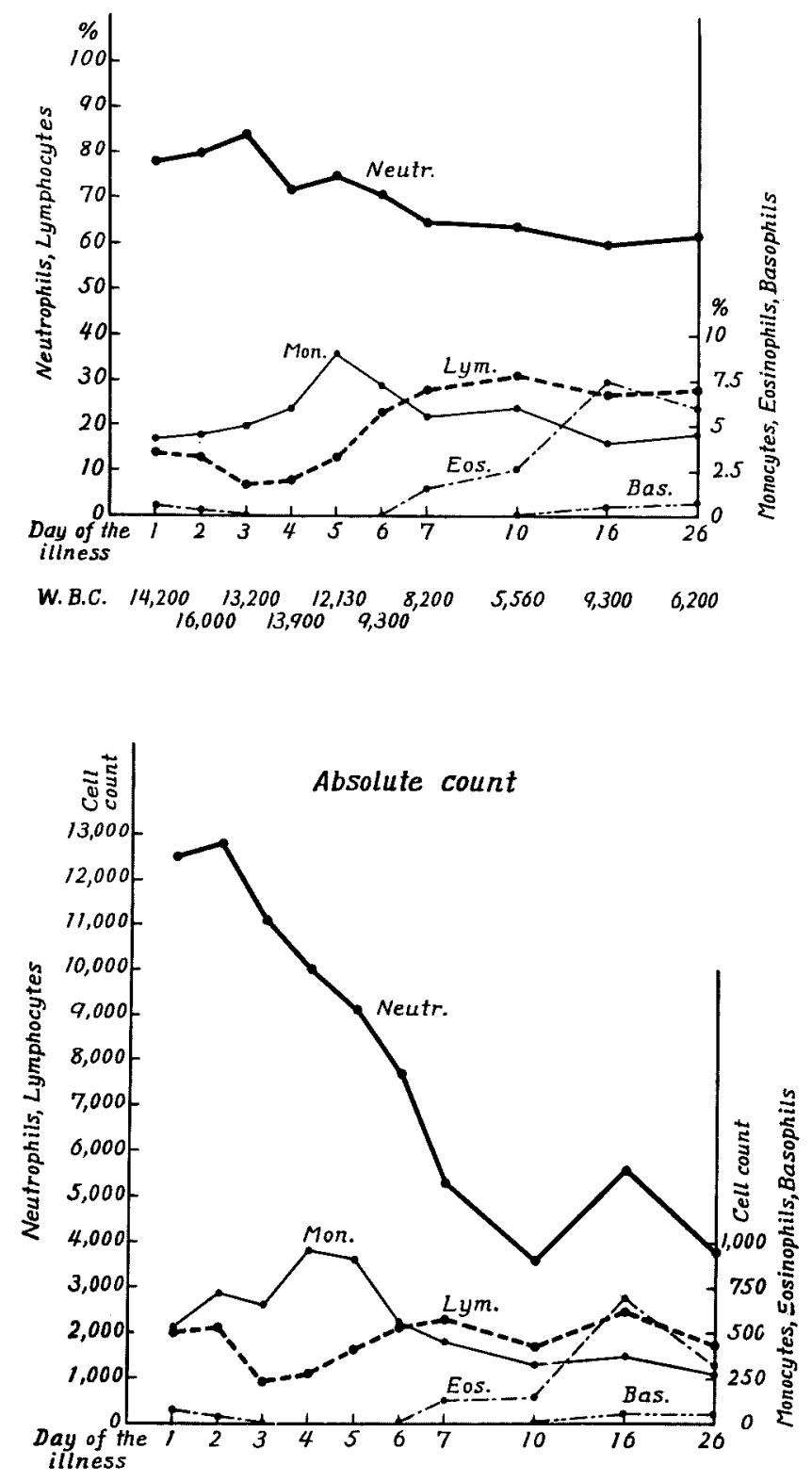

Fig. 2. Acute appendicitis, age $17 \AA$. 
the strength of the antibody-antigen reaction parallels that of the biological activity of antigen, but does not necessarily parallel the change in the absolute number of antibody-producing cells.

2. Comparison of Absolute Count and Percentage of the White Blood Cells in Infectious Diseases

As stated above, it was found that the strength of the cell-bacterium reaction depends on the absolute count of leucocytes, but that of the antibody-antigen reaction depends on the quality of the proliferating cells. This observation may become clear when we make comparison of the curves plotting the absolute numbers of the leucocyte constituents with the curves of their percentages, as follows.

1) Leucocytotic diseases

a) Acute appendicitis (Fig. 2)

In percentage, the typical form of leucocytotic diseases is manifest in leucocyte reaction here. The rise and fall in the percentages of various kinds of leucocytes in the stages of struggle, defense and convalescence beautifully reflect the characteristic changes in the hematopoietic phases of the bone marrow and the fields of blood denfense reaction, the neutrophils and the lymphocytes increasing and decreasing antagonistically, showing a regular typical change. In the chart of absolute numbers, a steep rise in the count of neutrophils is conspicuously obvious, but the lymphocytes show only a slight decrease on the 3rd-5th days of the disease, remaining in the normal range of $2,000-2,500$ in absolute number throughout, the host-parasite relation being not at all disclosed.

b) Scarlet fever (Fig. 3)

In percentage, the graph shows the typical pattern of leucocytotic diseases, as in the above, the changes in neutrophils and lymphocytes describing a pair of gentle antagonistic curves, clearly reflecting the adaptation of the host's internal environment. In the graph of absolute counts, however, the neutrophils show a very remarkable change, while the lymphocytes begin to increase rather steeply on the 7th day, to reach the peak of 6,100 on the 10th day and then to drop off gradually to the normal level. The absolute counts of leucocytes of various kinds show mutually independent diverse change, nowhere reflecting a definite biological trend.

2) Normo-leucocytotic diseases (Fig. 4)

In the percentage graph, we see the typical pattern of change in the diseases wherein the total leucocyte count does not rise or drop beyond the normal range ${ }^{3)}$. The peaks in the curves of neutrophil, monocyte, lymphocyte, eosinophil and basophil counts appear in the order named, and the entire chart shows very wellregulated biological curves.

In the chart of absolute counts, these curves are distorted beyond recognition 


\section{Percentage}
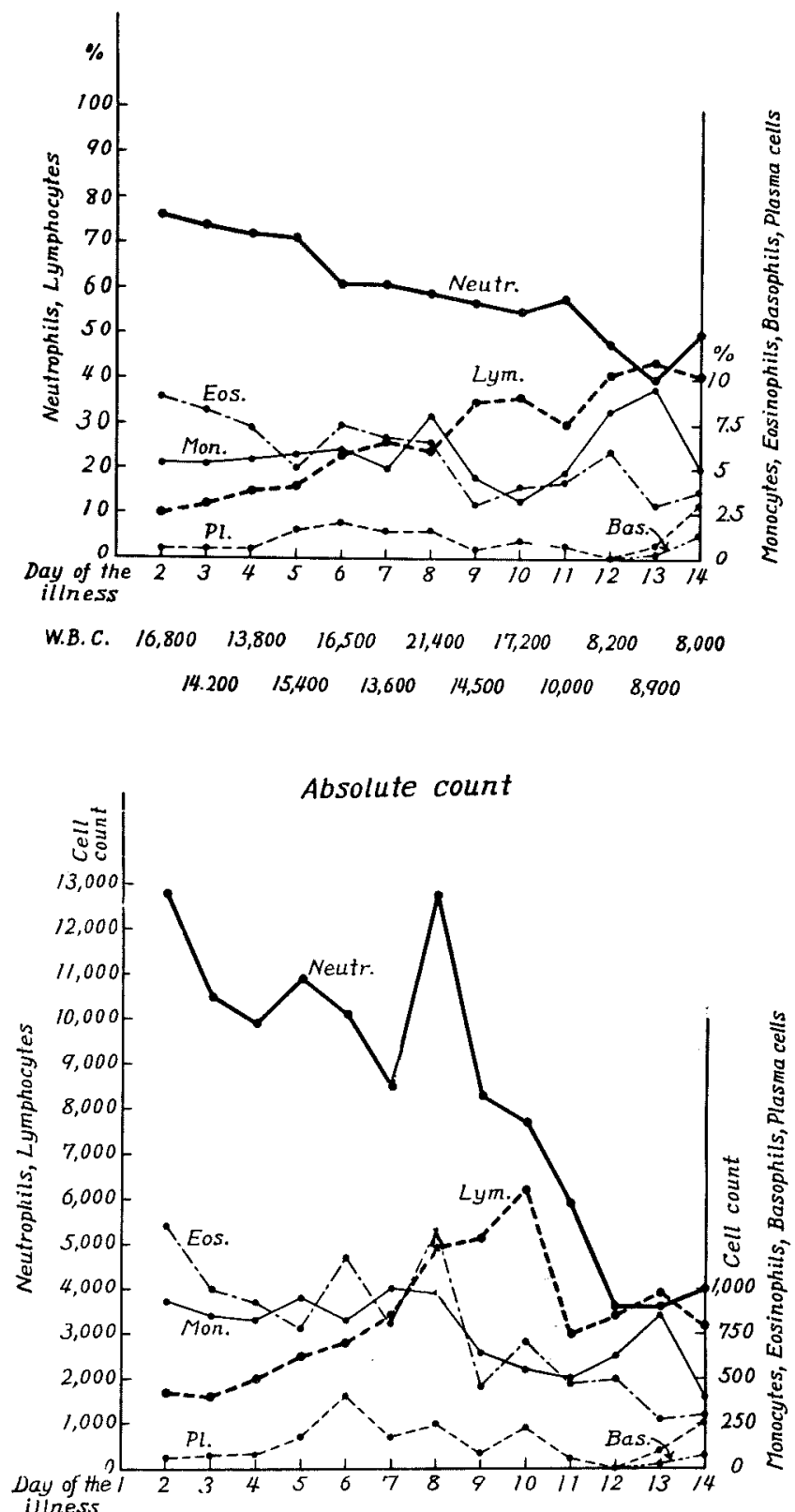

Fig. 3. Scarlet fever (38 cases). 

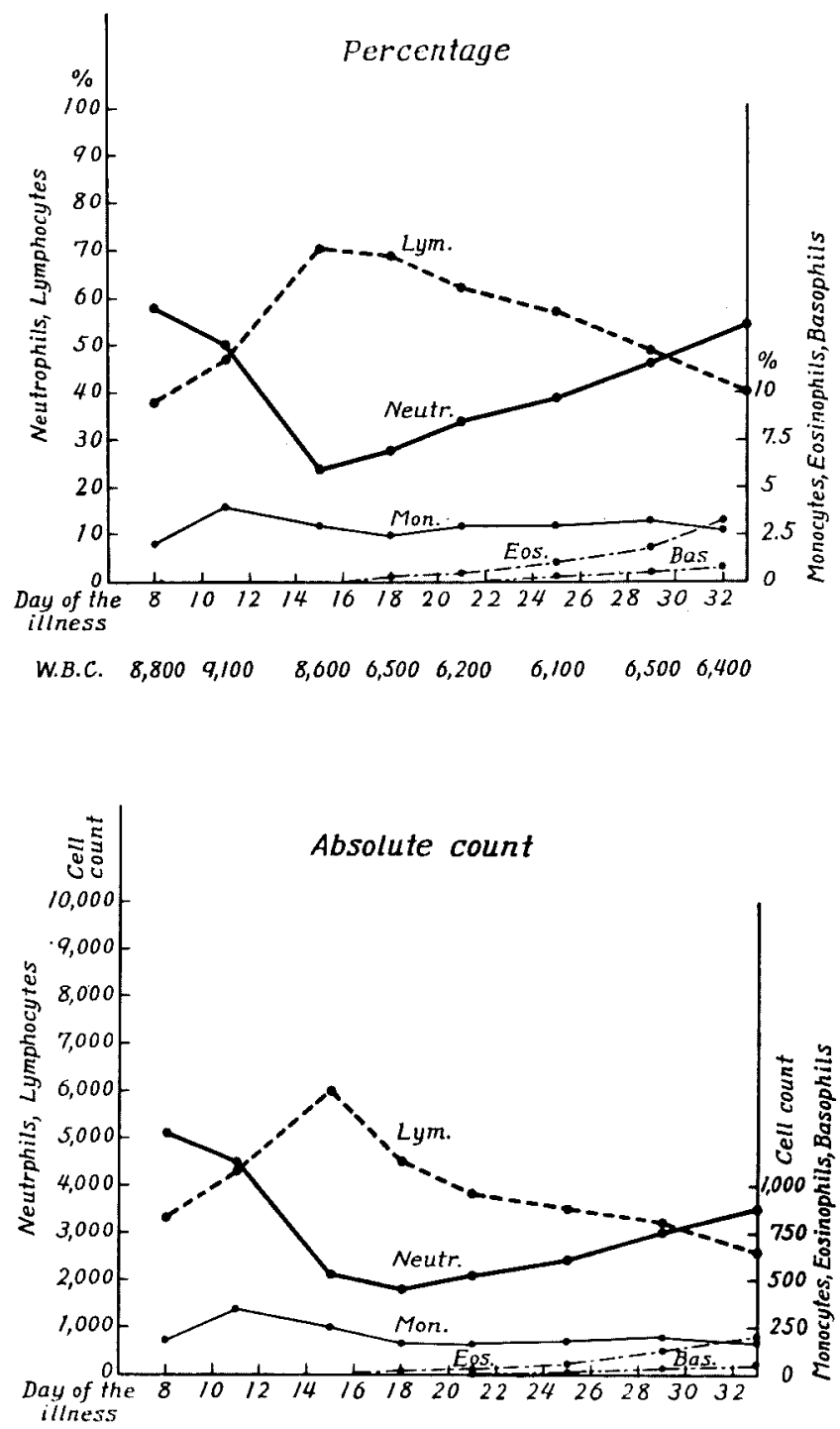

Fig. 4. Glandular fever, age, 26 占.

of the antagonistic relation between neutrophils and lymphocytes, but in these diseases, the numerical change of neutrophils is smaller than in leucocytotic diseases, showing no such vehement ups and downs as in the latter, so that the two charts in Fig. 4 show close resemblance.

3) Leucopenic diseases (Fig. 5)

In the chart of percentages, the curves follow the pattern typical of leucopenic 

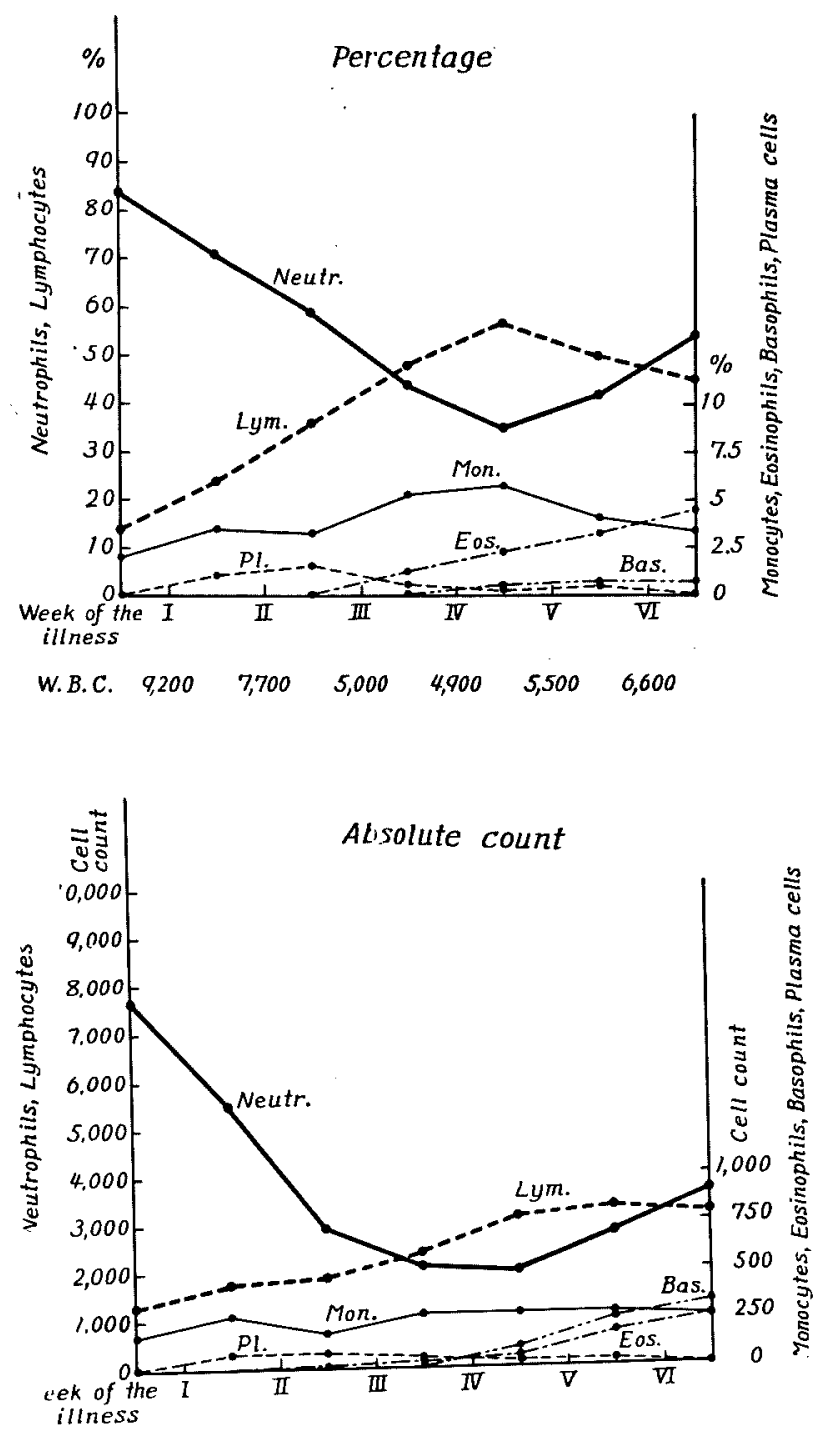

Fig. 5. Typhoid fever (536 cases).

diseases, the antagonistic relation of neutrophils and lymphocytes, the mutual relation among the various kinds of leucocytes, the relation between the hematopoietic phases of the bone marrow and the fields of blood defense reaction and the host's adaptation to the changing internal environment being clearly reflected. In the chart of absolute counts, however, the neutrophil count is exceptionally notable, decreasing very perceptibly in the acme stage, while the 
lymphocyte count falls at the outset and then rises again to the normal level in the 3rd week of disease and even further later on, but the counts of the other miscellaneous cells tend to be confined in the narrow range of below 500, the poverty of such cells being accentuated, and the chart reminds us of the picture of Naegeli's insufficiency of the bone marrow ${ }^{34}$.

The above comparative study reveals that the chart of percentages of leucocytes of various kinds serves in clearly representing the biological significance of the author's so-called hematopoietic phases, fields and times of defense reaction and in reflecting the host's adaptation to the changes of internal environment, while the chart of absolute counts, reflecting only the number of individual cell types but not the change in the quality, may serve in indicating the strength of the cell-bacterium reaction, but not at all the strength of the antibody-antigen reaction.

For further elucidation of the relative merits of percentage and absolute count in discussing the leucocyte reaction, we should consider the body's defense mechanism and study the essential nature and distribution of defense energy.

\section{Law of Conservation of Defense Energy}

The author, in the study on the essential nature of hematopoiesis in the bone marrow, laid stress on the adaptation of the body to internal environment, especially on the distribution of defense energy of the host mobilized in the host-

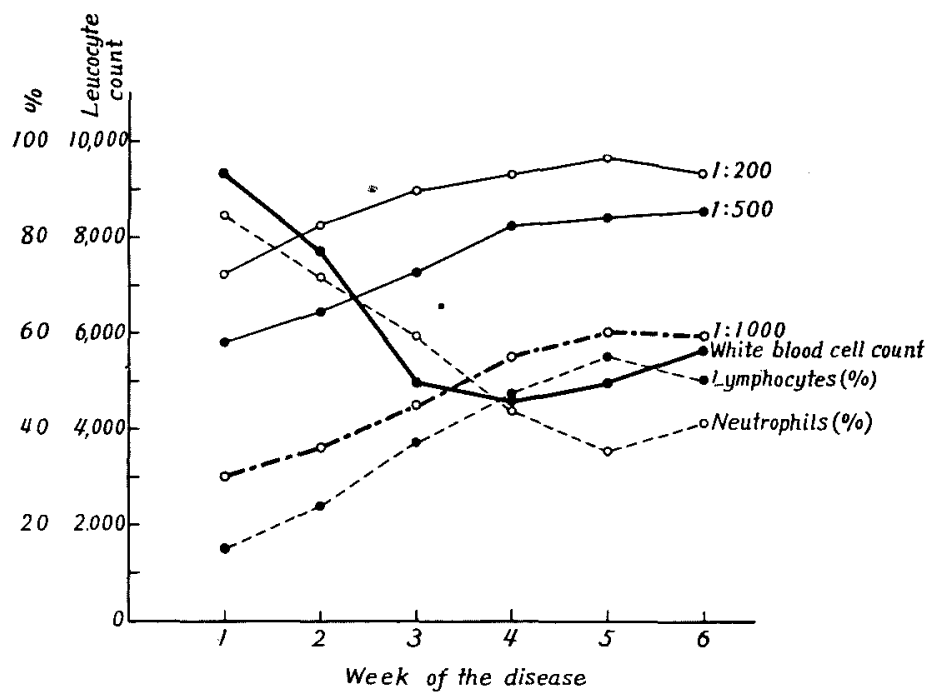

Fig. 6. Antagonistic-constant relation between the defense force of blood cells (cell-bacterium reaction) and that of blood serum (antibodyantigen reaction) in 536 typhoid cases. 
parasite struggle.

In the effort for adaptation of the host body to the internal environment, the defense force of blood in the host-parasite relation as stated in a previous report ${ }^{2)}$, consists of the defense force of blood cells and that of blood serum, acting antagonistically as shown in Fig. 6. For example, in leucopenia in typhoid fever, the percentage of neutrophils and the antibody (agglutinin) titre in serum rise and fall inversely to each other, the weakening in the defense force of blood cells (phagocytosis) due to the decrease of neutrophils being made up by the strengthening serum defense force due to the rise of antibody-production in the serum.

The rise in the antibody titre runs parallel to the increase in percentage of lymphocytes, revealing that the neutrophils, the main combatants in the field of cell-bacterium reaction, act in antagonism to the lymphocytes, the principal fighters in the field of antibody-antigen reaction, and vice versa.

This antagonism is a very important relation in disclosing the distribution of defense energy in the host body. The decline in the cell-bacterium reaction due to leucopenia (hitherto described as Naegeli's so-called insufficiency of the bone marrow ${ }^{34}$ ) is backed up by an increase of antibody production, which now takes over the main defense reaction, suggesting that life is preserved most rationally and purposefully.

As every natural phenomenon or reaction is enacted by give and take of energy, the above reaction must be attributed to the quantitative relation of the defense energy mobilized by the host in the fields of cell-bacterium reaction and antibody-antigen reaction.

The decrease in the energy poured into the cell-bacterium reaction field is made up by an equal increase in the energy expended in the antibody-antigen reaction field and vice versa, that is, the total energy released into the two fields is always constant.

Now, denote the energy mobilized in the field of cell-bacterium reaction by $\mathrm{C}$ and that mobilized in the field of antibody-antigen reaction by $A$, and the total defense energy mobilized $(\mathrm{E})$ is represented by

$$
\mathrm{E}=\mathrm{C}+\mathrm{A} \ldots \ldots \ldots \ldots \ldots \text { Constant }
$$

Thus, we have arrived at the law of conservation of total defense energy to the effect that the sum total of the energy expended in the two fields of cellbacterium reaction and antibody-antigen reaction in the host-parasite relation is constant and equal to the sum total of the host's defense energy (provided that the host's functions are not impaired).

This law is comparable to the law of conservation of mechanical energy, providing that the sum of kinetic energy $(\mathrm{T})$ and the potential energy $(\mathrm{U})$ in a system closed to external force is equal to the mechanical energy (E) and remains always constant. It has the following corollaries; 


$$
\mathrm{E}=\mathrm{T}+\mathrm{U} \ldots \ldots \ldots \ldots \ldots \text { Constant }
$$

(2) The sum of the energy released in the cell-bacterium reaction and that in the antibody-antigen reaction, that is, the total defense energy of a host body, is not increased or decreased by a difference of the stimulant factor.

(3) The strength of the cell-bacterium reaction and that of the antibodyantigen reaction are in a constant-antagonistic relation.

Accordingly, the cell-bacterium reaction and the antibody-antigen reaction in the defense mechanism of the host body, as shown in Fig. 7, are in antagonisticconstant relation, as are the sympathetic and the parasympathetic nerve systems in the autonomic nervous system.

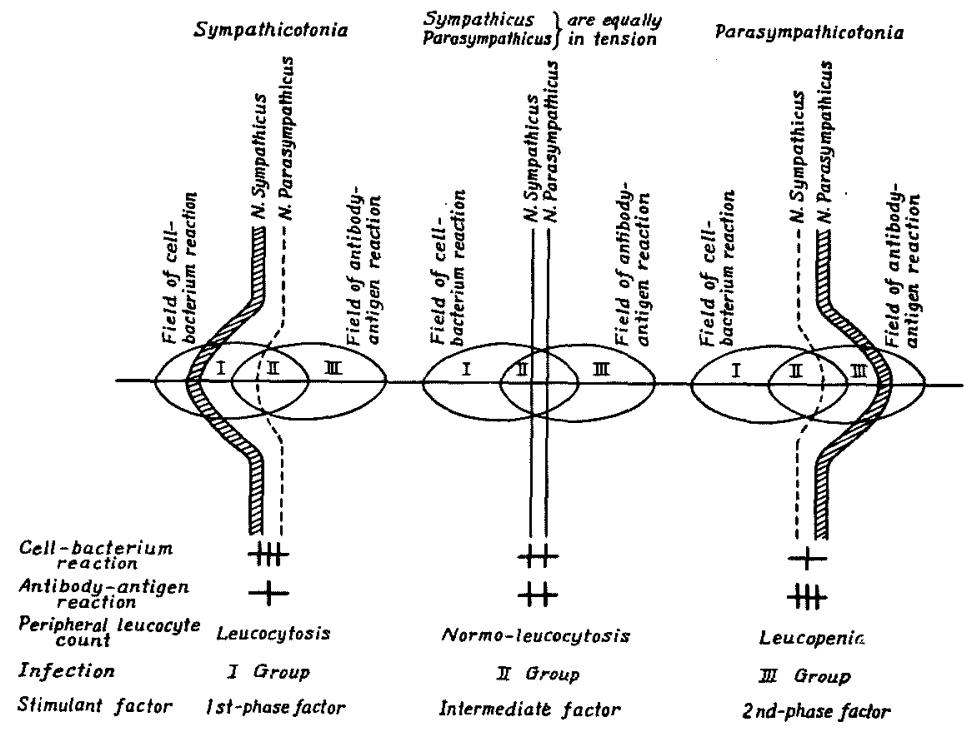

Fig. 7. Control mechanism of the autonomic nervous system over the fields of cell-bacterium reaction and antibody-antigen reaction.

As above, the law of conservation of defense energy is in perfect harmony with the law of conservation of energy independently propounded by Mayer ${ }^{35}$ ) in 1842 (deduced from the observation of the oxygen-metabolism process in erythrocytes), Joule $^{36}$ ) in 1843 (deduced from the behavior of electric energy) and Helmholtz ${ }^{37}$ ) in 1847 (deduced physico-mathematically), respectively.

The allocation of defense energy of the host body is governed by the autonomic nervous system in and after the acme stage, acting specifically according to the variety of the pathogenic factors ${ }^{27}$. As shown in Fig. 7, when the stimulant factor is of the Ist phase, sympathicotonia is induced and the main part of the defense energy is sent into the cell-bacterium reaction field; when it is 
of the 2nd phase, parasympathicotonia is evoled and the main part of the defense energy is allocated to the antibody-antigen reaction field, and when the factor is of the intermediate type, both the sympathicus and the parasympathicus are equally in tension and the defense energy is thrown equally into both the defense reaction fields.

As the sympathetic and parasympathetic nerve systems act antagonistically to each other, the defense energy is distributed antagonistically in the two fields of defense reaction, that is, most rationally and purposefully, and the reason why the strength of the cell-bacterium reaction and that of the antibody-antigen reaction stand in antagonistic-constant relation is very obviously disclosed.

In this series of studies, the field of defense reaction is divided into the I, II and III regions, according to the characteristics and relative strengths of the cell-bacterium reaction and the antibody-antigen reaction, and the infectious diseases are divided into the I, II and III Groups. This tripartite system of division accentuates the classification of infectious diseases serviceable in the regions of basic, clinical and prophylactic medicine, as already detailed ${ }^{1-16}$. It is apparent that the essential characteristics of the 3 Groups have their origin in the essential nature of the cell-bacterium reaction and the antibody-antigen reaction and the quantity of energy released in the fields of the two reactions.

Accordingly, as shown in Fig. 8, the defense energy, the coordination mechanism and the defense mechanism, in their mutual correlation, justify the tripartite classification of infectious and inflammatory diseases applicable uniformly in basic, clinical and prophylactic medicine, though in appearance the clinical pictures seem to be multifariously confounded by the complex interaction of the various strength of the two reactions. The law of conservation of defense energy is a basic law of defense reaction shown by the host body in adaptation to the internal environment, and enables the host body to make the adaptation in a most rational and purposeful way and to preserve its life in the highest possible security.

Distribution of Defense Energy in Infectious and Inflammatory Diseases and the Relation between the Absolute Number and Percentage of Various Leucocytes in the Blood Picture

A Study on the biological significance of the absolute number and the percentage of the leucocytes of various kinds, as based on the above law of conservation of defense energy, is of very high importance from the angles of clinical medicine and biology.

1. Relation of Distribution of Defense Energy in the Fields of Cell-bacterium and Antibody-antigen Reactions to the Absolute Number of Various Leucocytes

The defense energy is allocated to the two reaction fields, according to the specificities of the stimulant factors. The defense energy for production of 


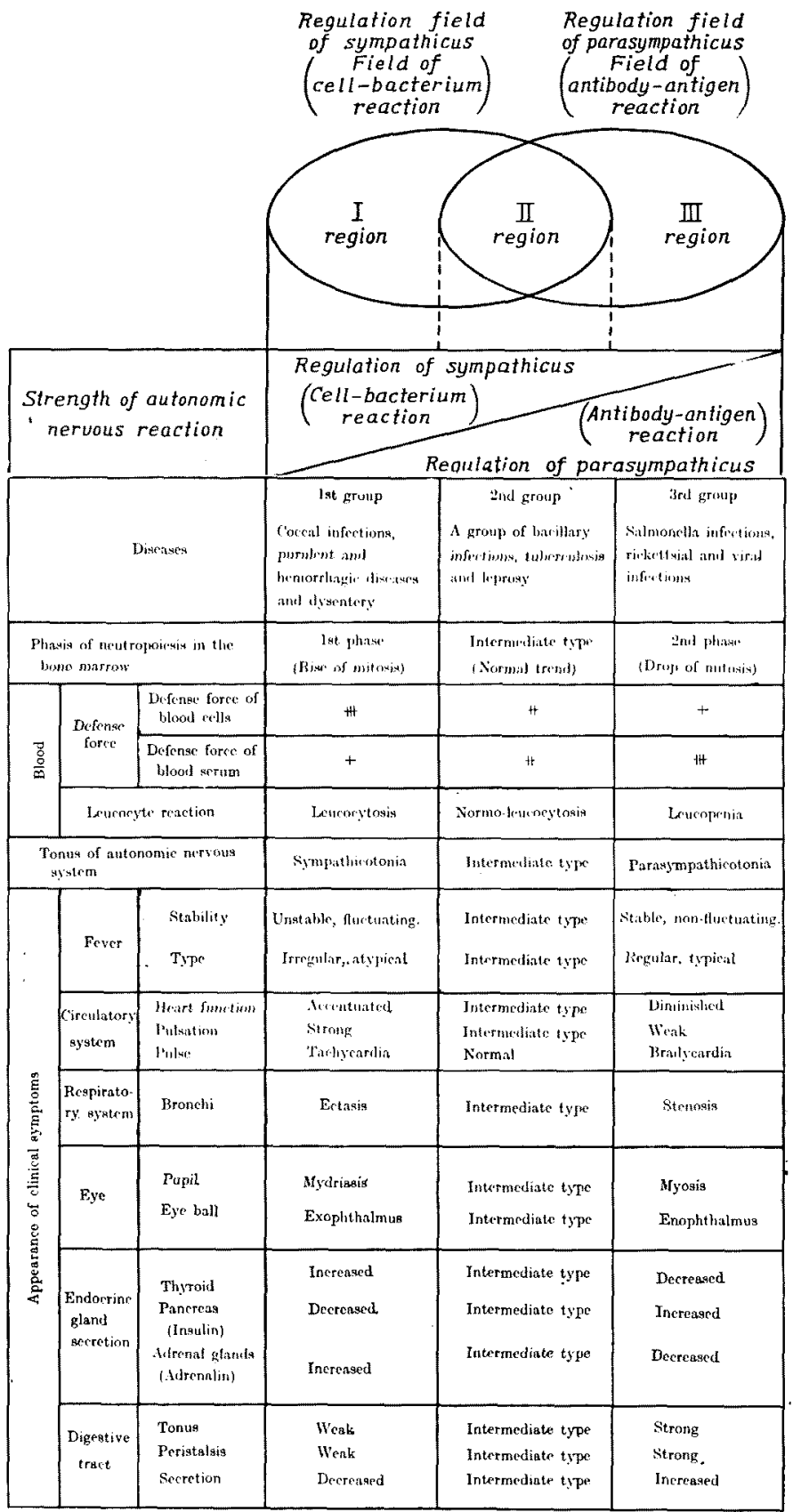

Fig. 8. Relationships between the defense energy, coordination mechanism and defense mechanism. 


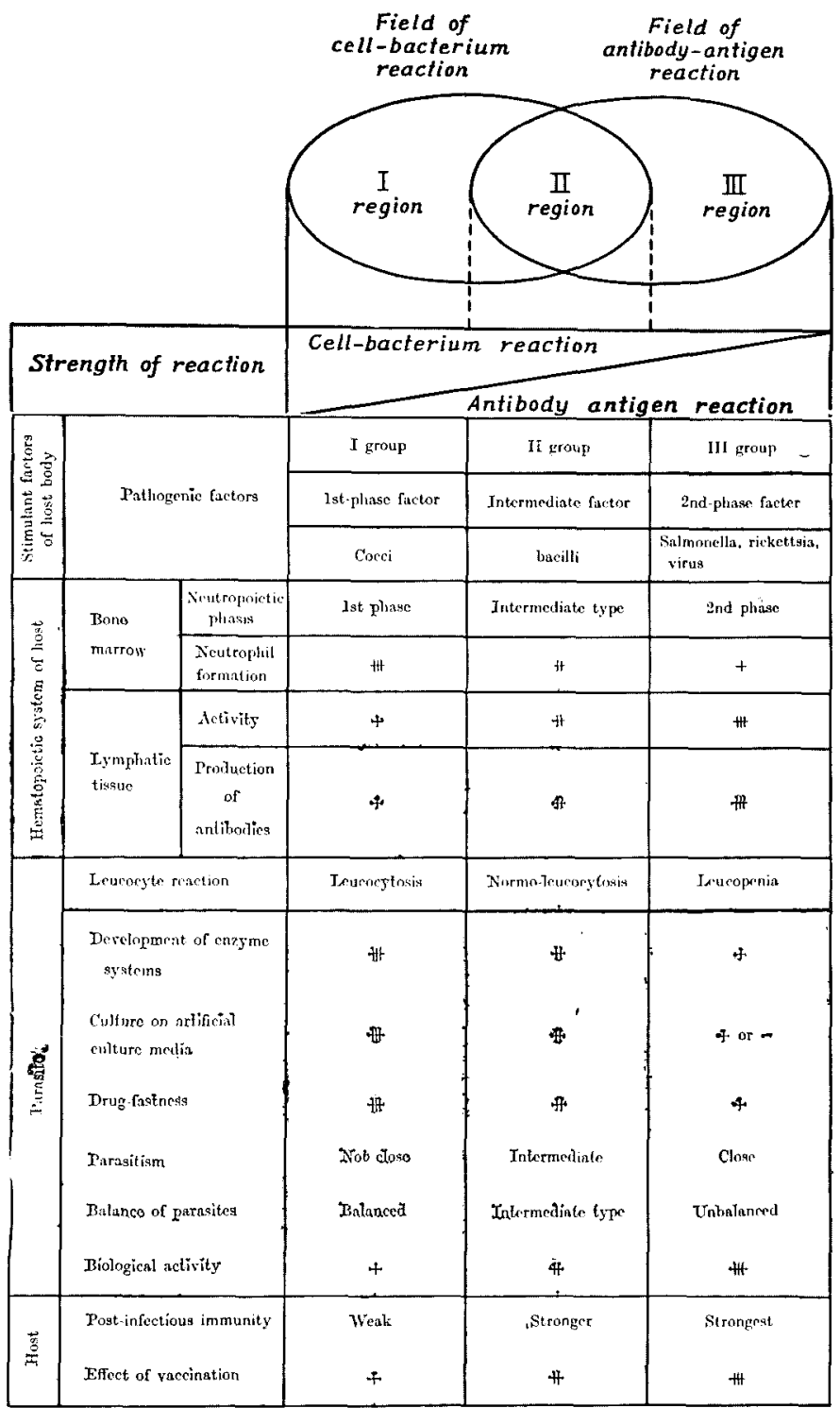

Fig. 9. Relationships between the specificity of pathogenic factors, and the neutropoietic system of the bone marrow and lymphatic system.

defense reaction cells is mainly allocated to the neutropoietic system of the bone marrow under stimulation by the 1st-phase factors, and to the lymphopoietic system acting antagonistic-coordinatively with the former under stimulation by the 2nd-phase factors, but equally to the two systems under stimulation by 
intermediate factors. As the results, the mitosis of the neutropoietic system in the bone marrow shows a diphasity, either increasing or decreasing the output of neutrophils, but in the lymphopoietic system (lymphatic system) stimulation by 1st-phase, 2nd-phase and intermediate factors biologically activates the system in respectively different strength and induces generation of lymphocytes producing specific antibodies (Fig. 9).

Thus, the defense energy, changing in form and transmitted through material, causes increase and decrease of the cell count accentuated morphologically in neutrophils, and qualitative change accentuated functionally in lymphocytes, and finally changes into energy for killing the pathogenic agents, but the total quantity of defense energy does not change with the variety of the pathogenic factors.

The diagram of such allocation of defense energy is presented by the characteristics of neutrophils and lymphocytes.

1) Neutrophils and lymphocytes take up $55 \%$ and $38 \%$ on the average, respectively, of the total leucocytes in normal blood, but the two kinds are utterly different functionally. Their respective life spans stand in the ratio of $1: 1.5$ or more, so that we may deduce that the reaction power of both kinds is nearly equal and the two kinds stand in antagonistic equilibrium.

2) The neutrophil count in normal blood is about 4,500, but in infectious diseases it changes widely in the range of $300-40,000$ and sometimes even to 60,000 , but the lymphocyte count, around 2,500 in normal cases, remains within the range of $1,000-6,000$ in infectious diseases, scarcely falling below 1,000 or rising above 6,000. Thus, neutrophils increase and decrease over the extremely wide range of 15/1-1/15 of the normal count while the count of lymphocytes rises and falls in the much narrower range of $2 / 1-1 / 2$.

This wide disparity in the range of numerical change is due to the essential differences of phagocytosis (cell-bacterium reaction) and antibody-production (antibody-antigen reaction), the strength of phagocytosis depending on the total number of neutrophils, and that of antibody production on the functional activity of lymphocytes (Figs. 2-5).

So, neutrophils and lymphocytes are two entirely different types of cells, both numerically and functionally, but between themselves, they make up $93 \%$ (in percentage) of leucocytes, so that in infectious and inflammatory diseases, the rise and fall in neutrophil count is predominant cause determining the total leucocyte count, as observed in leucocytotic, normo-leucocytotic and leucopenic types of diseases.

In infectious diseases, neutrophils show marked numerical change but little quantitative change, except a nuclear shift to the left, while lymphocytes show little numerical but marked functional change. Therefore, when the absolute num- 
ber is taken as the criterion, the number of neutrophils and other phagocytes will reflect the strength of the cell-bacterium reaction, but the number of lymphocytes and such antibody-producing cells can not be an index of the strength of the antibody-antigen reaction, since the number is not related to the functional activity of lymphocytes.

2. Relation of Distribution of Defense Energy in the Fields of Cell-bacterium and Antibody-antigen Reactions to the Percentage of Various Leucocytes

The law of conservation of defense energy indicates that the defense energy is distributed rationally and purposefully in both fields of cell-bacterium reaction and antibody-antigen reaction.

Accordingly, it can be easily understood that, when the sum total of various kinds of leucocytes in every stage of infectious and inflammatory diseases is taken as 100 in number, the percentages of the various kinds of leucocytes show the distribution of defense energy (Figs. 2-5).

That is, the percentages of various kinds of leucocytes indicate the present state of adaptation of the host body to the internal environment. When chronologically observed, the percentages are traced very systematically, as reported in Report $6^{6}$ ), and 3 basic types of biological leucocyte curves will appear, which are characteristic for leucocytotic, normo-leucocytotic and leucopenic diseases, showing clearly the distribution of defense energy in every stage.

3. On the Clinical Value of Absolute Count and Percentage of Leucocytes

It has been made clear that, in infectious and inflammatory diseases, a table of percentages of the various kinds of leucocytes reflects most accurately the state of the host's adaptation to the internal environment. A comparative study of the clinical values of absolute counts and percentages of leucocytes of various kinds leads to the following conclusions.

1) As the absolute counts have to be computed from the percentage and the total leucocyte count, it takes an additional operation.

2) The percentages are represented in simple numbers below 100, but the absolute counts are usually more unwieldy numbers.

3) The percentages very obviously reflect the distribution of defense energy, but in a chart of absolute counts, the increase and decrease of phagocytes such as neutrophils is very conspicuous, so that the distribution of defense energy is hard to perceive.

4) The percentages clearly suggest the functional relation between the different kinds of leucocytes, the antagonism between neutrophils and lymphocytes, and the times when the different kinds of leucocytes come into reaction, but a chart of absolute counts does not make such points obvious.

5) The percentages indicate the fields of main defense reaction but as abso- 
lute counts indicate only the change in counts of individual kinds of leucocytes, the field of main defense reaction is obscure.

6) The percentages serve in tracing biological leucocyte curves, which form regular patterns characteristic to the 3 groups of diseases of leucocytotic, normoleucocytotic and leucopenic types, but with absolute counts, only the curves of neutrophils and such phagocytes show very conspicuous ups and downs, giving irregular patterns devoid of any conformity to types.

7) The percentages indicate the antagonistic relation between the neutropoietic system of the bone marrow and lymphatic system, but the absolute counts do not.

8) The percentages clearly bring out the relative strength of the cellbacterium reaction and the antibody-antigen reaction, but the absolute counts do not.

9) The percentages clearly show the present state of adaption of the host body to the internal environment and suggest that they are of high clinical value in facilitating identification of the stimulant factor and prediction of further progress and prognosis. The overconspicuousness of increase or decrease of neutrophils (phagocytes) in a chart of absolute counts tends to mask the change in the antibody-producing cells, so that it is difficult to grasp how the host body is progressing in the process of adapting to the internal environment.

The above findings make it clear that the percentages are more serviceable than the absolute counts in discussing the changes of the various kinds of leucocytes. In cases of special necessity, say, insufficiency of the bone marrow, the absolute count can be readily computed by the total leucocyte count multiplied by percentage. In short, the percentages are clinically of very high value, and indication of the absolute count is rarely required.

Naegeli ${ }^{34}$ ) showed the curves of absolute counts of leucocytes, but Schilling ${ }^{38}$ ) published curves of percentages, called Schilling's biological leucocyte curves. As already pointed out, Naegeli and Schilling flourished in the age when no one thought of the monophasic formation of erythrocytes and the diphasic formation of neutrophils in the bone marrow. Moreover, the one field reaction of erythrocytes, the two field reaction of neutrophils and lymphocytes under the antagonistic relation of the two, that is, the basic notions of hematopoietic phases in the bone marrow, fields and times of blood defense reaction and the law of conservation of defense energy were still lying undiscovered; so, it is quite natural that the absolute counts of the various kinds of leucocytes were preferred as the most valuable indices.

Schilling, however, upon profound study of the blood picture in infectious diseases, empirically recognized the higher serviceability of percentages over that of absolute counts of the various kinds of leucocytes and arrived at his biological leucocyte curves, as Heilmeyer ${ }^{39}$ ) has already pointed out. 


\section{On Counting Nucleated Cells in the Bone Marrow}

As the counting method of various kinds of leucocytes is of important in discussing the essential nature of the blood defense reaction, so the method of counting and expressing the various kinds of nucleated cells in the bone marrow becomes important in investigating the hematopoietic function of the bone marrow.

The method of counting such nucleated cells in current use in Japan consists in counting all the nucleated cells of the erythoropoietic, leucopoietic, reticuloendothelial and megakaryopoietic systems, and computing the percentages of individual kinds to the total count. But $\mathrm{Rohr}^{40)}$ took 500 cells of the leucopoietic system, including lymphocytes in the bone marrow, and computed the percentages by types of cells, while he counted separately the nucleated cells of the erythropoietic and reticuloendothelial systems and the megakaryocytes, coming into the field of vision, and computed the percentages of these cells to 100 of leucocytes and lymphocytes. He claimed the high rationality of this so-called Rohr's counting method on the following grounds:

1) This method facilitates comparison between the bone marrow picture and the peripheral blood picture, and helps very much in estimating the maturation of leucocytes and the velocity of their flow from the bone marrow into the peripheral blood.

2) The cells of the leucopoietic system are evenly distributed in the bone marrow, but the erythroblasts are often found focally aggregated like nests in some parts, so that the fluctuation of the erythroblast count is very large. Reticuloendothelial cells are also prone to such uneven distribution, and, according to Krumbhaare \& Custer ${ }^{41)}$, such a tendency to aggregation is still stronger in megakaryoblasts.

According to Rohr's reports, the ratio of erythroblasts to granulocytes (leucocytotic cells minus lymphocytes), computed according to this method, stands at 3: 1 in normal human subjects; this ratio is said to be an important ratio showing the relation between the erythropoietic system and the leucopoietic system in the bone marrow.

In practice, Rohr's method is convenient and rational in estimating the formation and maturation of blood cells in the bone marrow and the velocity of their efflux into the peripheral blood, as follows:

1) In a normal man, erythroblasts and reticuloendothelial cells do not flow out from the bone marrow into the peripheral blood, except in a few acute febrile infectious diseases including relapsing fever, eruptive typhus and Japanese encephalitis, in which a few erythroblasts comes out into the peripheral blood in the extremely low frequency of $1-3$ among 500 leucocytes $(0.2-0.6 \%)$.

In the bone marrow, however, erythroblasts are found usually in the ratio of 27-30 per 100 leucocytic series cells, and reticuloendothelial cells (including plasma 
Table I. Normal Human Myelogram

\begin{tabular}{|c|c|c|c|c|c|c|c|c|}
\hline \multirow{3}{*}{ 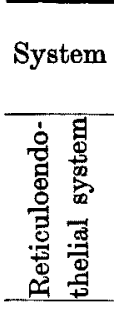 } & \multirow{2}{*}{\multicolumn{3}{|c|}{ Cells }} & \multicolumn{3}{|c|}{ Saito \& Shimoyama } & \multirow{2}{*}{\multicolumn{2}{|c|}{ Rohr }} \\
\hline & & & & Maxim.- minim. & Aver & ge & & \\
\hline & \multicolumn{3}{|c|}{$\begin{array}{l}\text { Lymphoid reticulum cells } \\
\text { Plasmacellular reticulum cells } \\
\text { Macrophges }\end{array}$} & $\begin{array}{l}4-1 \\
4.4-0.8 \\
0.1-0\end{array}$ & $\begin{array}{c}2.5 \\
2.5 \\
0.1-0\end{array}$ & 5 & $\begin{array}{l}6-1 \\
2 \\
0\end{array}$ & 8 \\
\hline 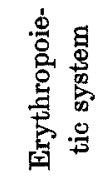 & \multicolumn{3}{|r|}{$\begin{array}{l}\text { Basophil } \\
\text { Polychromatic } \\
\text { Oxyphil }\end{array}$} & $\begin{array}{r}5.2-1.2 \\
32.1-13.8 \\
8.1-1.5\end{array}$ & $\begin{array}{c}3 \\
20 \\
4.4\end{array}$ & 27 & $\begin{array}{r}5 \\
9 \\
16\end{array}$ & 30 \\
\hline \multirow{4}{*}{ 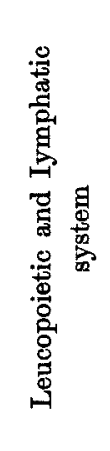 } & \multicolumn{3}{|c|}{ Myeloblasts } & $2.5-1.0$ & 2 & & 1 & \\
\hline & \multirow{2}{*}{ 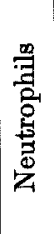 } & Myelocytes & $\begin{array}{l}\text { Unmatured } \\
\text { Halt-matured } \\
\text { Matured }\end{array}$ & $\begin{array}{r}3.8-4.1 \\
7.5-4.1 \\
13-8.9\end{array}$ & $\begin{array}{r}2.6 \\
6.4 \\
11.2\end{array}$ & & $\begin{array}{l}4 \\
6 \\
7\end{array}$ & \\
\hline & & \multicolumn{2}{|c|}{$\begin{array}{l}\text { Metamyelocytes } \\
\text { Rod-shaped } \\
\text { Segmented }\end{array}$} & $\begin{array}{l}14.8-9.8 \\
39.1-27.3 \\
22-6.8\end{array}$ & $\begin{array}{l}12.2 \\
31.6 \\
11.9\end{array}$ & 100 & $\begin{array}{r}9 \\
41 \\
17\end{array}$ & 100 \\
\hline & \multicolumn{3}{|c|}{$\begin{array}{l}\text { Basophils } \\
\text { Eosinophils } \\
\text { Monocytes } \\
\text { Lymphocytes }\end{array}$} & $\begin{array}{l}0.5-0 \\
7.8-2.6 \\
4-1 \\
20.3-9.2\end{array}$ & $\begin{array}{r}0.2 \\
4.7 \\
2.8 \\
14.3\end{array}$ & & $\begin{array}{l}1-0 \\
4 \\
2 \\
11\end{array}$ & \\
\hline 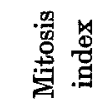 & \multicolumn{3}{|c|}{$\begin{array}{l}\text { Erythropoietic system \%o } \\
\text { Leucopoietic system \%o }\end{array}$} & $\begin{array}{r}43.1 \\
2.4\end{array}$ & & & $\begin{array}{l}42 \\
2.5\end{array}$ & \\
\hline
\end{tabular}

cells of perivascular origination), too, are found in the frequency of 5-8 against 100 leucocytic system cells (Table I).

So, the percentage of erythroblasts not coming out into the peripheral blood in proportion to the total count of nucleated cells in the bone marrow would be

$$
\begin{aligned}
& \frac{27}{100+27+5} \times 100=-20.4 \% \quad \ldots \ldots \ldots . \quad \text { Saito \& Shimoyama } \\
& \frac{30}{100+30+8} \times 100=22.2 \% \quad \ldots \ldots \ldots \text { Rohr. }
\end{aligned}
$$

As this $20.4-22.2 \%$ of erythroblasts do not appear in the peripheral blood, we may say there is a fault of $20.4-22.2 \%$ in the comparison of the bone marrow picture and the blood picture when using the usual counting method. As this fault is rather large in comparison to the amount of change in the function of the neutropoietic system of the bone marrow in infections and inflammations, the 
change in the leucopoietic function in leucocytotic, normo-leucocytotic and leucopenic diseases would fall into this fault and appear much smaller or disappear, leading to misestimation of the leucopoietic function, especially, the neutropoietic function.

2) The erythroblasts, as stated above, are often aggregated focally and unevenly distributed in the bone marrow.

3) In the bone marrow, erythrocytes are produced monophasically, but leucocytes, especially, neutrophils, the main constituents, are produced diphasically, in a quite different manner.

4) When the basis of computation of the nucleated cells of the leucopoietic system in the bone marrow is different from that in the peripheral blood, it is difficult to study the formation and maturation in the bone marrow and the outflow of leucocytes in comparison in the bone marrow and the peripheral blood.

The above reasons show the obvious unreasonableness of taking the total count of nucleated cells as the basis of computing. Rohr's counting method is a most reasonable one for it eliminates the above difficulties, and is very serviceable and clinically highly valuable for estimating the hematopoietic function of the bone marrow, not only in infectious and inflammatory diseases, but also in diseases of hematopoietic organs and in many other diseases.

In 1938, the present author began to apply Rohr's counting method in the study of the hematopoietic function of the bone marrow, and it is the application of this method that led to the discovery of hematopoietic phases in the bone marrow function, so that the present study owes very much to Rohr's method.

\section{CONCLUSION}

The present author has found that the defense reaction shown by the host in its effort for adaptation to the internal environment in infectious diseases is delopyed both in the field of cell-bacterium reaction and in that of antibodyantigen reaction. The question which of them is chosen as the field of main defense reaction depends upon the specificity of the pathogenic factor. The defense energy of the host is poured into these two fields, in proportion that is most rational and adapted to the purpose, and the characteristics of the I, II and III group diseases are brought out respectively.

Accordingly, the author has arrived at the law of conservation of defense energy to the effect that the sum of defense energy allocated to the two fields of cell-bacterium reaction and antibody-antigen reaction is constant and equal to the total defense energy.

This law is the same in implication as the law of conservation of energy of Mayer-Joule-Helmholtz. From the law are deduced the corollaries that the total defense energy is not changed by any difference of the pathogenic cause, and that the strength of the cell-bacterium reaction and that of the antibody-antigren 
reaction stand in antagonistic-constant relation. That is to say, the relationship between the cell-bacterium reaction and the antibody-antigen reaction in the the defense mechanism is entirely the same as that between the sympathicus and the parasympathicus in the autonomic nervous system in the coordination mechanism.

In discussing the relative biological significance of absolute count and percentage in the blood picture, we find that the neutrophils and such phagocytes, unspecific and without qualitative changes under different stimulatilon, show large numerical changes, while the lymphocytes and such antibody-producing cells, producing specific antibodies, show little numerical but large qualitative changes. Accordingly, the absolute count of phagocytes is an index of the strength of the cell-bacterium reaction, but the absolute count of antibodyproducing cells is not always an infallible index of the strength of the antibodyantigen reaction.

This fact leads to the conclusion that the absolute count of various kinds of leucocytes is of low biological value, as it is not serviceable in reflecting the distribution of defense energy.

The chart of percentages of various kinds of leuccocytes, on the other hand, clearly visualizes the allocation of defense energy in the two different fields of reaction, and indicates the characteristics of the stimulant factor, and thus, is of high biological value. Besides, in collation with the total count of leucocytes, when necessary, the absolute count can be obtained without difficulty, and the clinical value of such percentages is also high.

The method of counting the nucleated cells in the bone marrow in current use in Japan is the widely practiced method of counting all nucleated cells and computing the percentages of the cells of various kinds, but seeing that the formation and function of the cells of the 3 major divisions of the erythropoietic, the leucopoietic (including lymphocytes) and the reticuloendothelial (including the perivascular) system are entirely different and that the erythroblasts are often very unevenly distributed in the bone marrow, such a crude counting method is very irrational. It is also very inconvenient in making comparison of the leucocyte series in the bone marrow with those in the peripheral blood, to study the formation, maturation or velocity of outflow of leucocytes.

The counting method proposed by Rohr, giving due consideration to the radical difference of the 3 systems, is a decidedly more rational method, from the standpoint of the present author's idea of the hematopoietic phases of the bone marrow, the fields and times of blood defense reaction and the distribution of defense energy, and must be commended as being of high biological and clinical importance. 


\section{References}

(1) Saito, A., Tohoku J. exp. Med., 1961, 74, 309.

2) Saito, A., ibid., 1961, 74, 328.

3) Saito, A., ibid., 1961, 74, 339.

4) Saito, A., ibid., 1961, 74, 349.

5) Saito, A., ibid., 1961, 74, 366.

6) Saito, A., ibid., 1961, 74, 477.

7) Saito, A., ibid., 1962, 77, 28.

8) Saito, A., ibid., 1962, 77, 42.

9) Saito, A., ibid., 1962, 77, 56.

10) Saito, A., ibid., 1962, 77, 66.

11) Saito, A., ibid., 1962, 78, 166.

12) Saito, A., ibid., 1962, 78, 302.

13) Saito, A., ibid., 1963, 78, 410.

14) Saito, A., ibid., 1963, 80, 148.

15) Saito, A., ibid., 1963, 89, 268.

16) Saito, A., ibid., 1964, 82, 181.

17) Saito, A. \& Miyamoto, T., Acta haemat. Jap. (Jap.), 1959, 22, 1.

18) Saito, A., ibid., 1959, 22, 12.

19) Saito, A., ibid., 1960, 23, 619.

20) Saito, A., ibid., 1960, 23, 692.

21) Saito, A., ibid., 1960, 23, 816.

22) Saito, A., ibid., 1961, 24, 22.

23) Saito, A., ibid., 1961, 24, 453.

24) Saito, A., ibid., 1961, 24, 564.

25) Saito, A., ibid., 1961, 24, 674.

26) Saito, A., ibid., 1962, 25, 90.

27) Saito, A., ibid., 1962, 25, 201.

28) Saito, A. \& Ono, T., Tohoku J. exp. Med., 1958, 67, 253.

29) Saito, A. \& Shimoyama, K., ibid., 1958, 67, 263.

30) Saito, A. \& Miyamoto, T., ibid., 1958, 67, 341.

31) Saito, A., ibid., 1958, 68, 29.

32) Saito, A., ibid., 1958, 68, 151.

33) Selye, H., Textbook of Endocrinology, Acta Endocrinologica Inc., Montreal, Canada, 1949 , p. 837.

34) Naegeli, O., Blutkrankheiten u. Blutdiagnostik, 5 Aufl., Jurius Springer, Berlin, 1931, p. $235,239,589$.

35) Mayer, J.R., Iwanami Igakuziten (Jap.), Iwanami Co., Tokyo, 1953, p. 1296.

36) Joule, J.P., ibid., 1953, p. 638.

37) Helmohlotz, L.F., ibid., 1953, p. 1246.

38) Schilling, V., Das Blutbild $u$. seine Klinische Verwertung, $1 \mathrm{X}-\mathrm{X}$ Aufl., Gustav Fischer, Jena, 1933, p. 284.

39) Heilmeyer, L. \& Begemann, H., Hanbd. d. inn. Med., 4. Aufl, II Bd., Blut u. Blutkrankheiten, Springer, Göttingen, Heidelberg, 1951, p. 535.

40) Rohr, K., Das menschliche Knochenmark, Georg Thieme, Leipzig, 1940, p. 73.

41) Krumbhaare \& Custer, cited by Rohr, 40) p. 76. 